\title{
Nirogi Maatha: a timely investment for a healthy future generation in Sri Lanka
}

\author{
N Hemachandra ${ }^{1}$, C N Wijeyaratne ${ }^{2}$ \\ Sri Lanka Journal of Diabetes, Endocrinology and Metabolism 2014; 4: 60-61
}

Pregnancy is a 'stress test' for the carbohydrate metabolism of all mothers-to be. This is due to a significant increase in anti-insulin action by the placental hormones. The resulting increase of maternal insulin resistance requires a high compensatory rise of maternal insulin secretion; which when inadequate to meet the demand, manifests as gestational diabetes (GDM). Diabetes in pregnancy can be broadly divided into pre-gestational or type 2 diabetes (T2DM) and GDM. Although two distinct clinical conditions, they are a part of the continual process towards the development of T2DM and cardiovascular disease (CVD) risks in the lifecycle of a woman. Therefore GDM is now viewed as a good predictor of future diabetes in a given population. Furthermore, the offspring of mothers with poorly controlled diabetes are at greater risk of obesity and premature metabolic derangement (an intergenerational effect).

The prevalence of T2DM among our population has shown an exponential rise in the past few decades, with the age of manifestation becoming lower and with no major gender difference. The incidence of GDM has also increased exponentially, and clearly requires universal screening during pregnancy. Additionally, those identified as at high metabolic risk, require lifelong follow up beyond pregnancy and the post partum period.

Sri Lanka has a high quality primary care model for Maternal and Child Health (MCH) services under the leadership of the Family Health Bureau of the Ministry of Health. Therefore optimizing screening and management of GDM and preventing post partum progression to T2DM has implications well beyond MCH. Proper management of this "window of opportunity" for the prevention of diabetes is of extreme importance and must be viewed as a good health investment.

Gestational diabetes mellitus (GDM) is defined as carbohydrate intolerance of any degree with onset or first recognition during pregnancy, irrespective of whether dietary modification alone or in combination with insulin is required (ADA 1999).
This broad definition does not distinguish between previously undetected T2DM from pregnancy related true gestational diabetes that occurs when placental hormone levels peak in mid trimester.

Screening in early pregnancy (at antenatal booking) helps distinguish between these metabolic conditions, while post partum screening both in the short term and long term would help towards a more pragmatic follow up of the mothers and families at risk.

Therefore, the Diabetes Prevention Task Force of the Sri Lanka Medical Association (SLMA), along with all stakeholders ranging from health policy makers and planners in the Ministry of Health and practicing clinicians and academics from the fields of Internal Medicine, Endocrinology, Obstetrics, Paediatrics, Community Medicine and Family Medicine identified the importance of a novel initiative called Nirogi Maatha within the Nirogi Lanka Project - phase II carried out by the SLMA. This effectively targets diabetes in pregnancy with a life cycle approach that is expected to have a health impact on a cross generational basis.

Capacity building of the MCH field staff is therefore a priority in such an endevour. Therefore it is an essential pre-requisite to arrive at a consensus on a nationally applicable Clinical Practice Guideline for managing maternal diabetes during pregnancy. This was coordinated effectively by the Family Health Bureau in conjunction with the Sri Lanka College of Obstetricians and Gynaecologists, Ceylon College of Physicians, Endocrine Society of Sri Lanka and the Nirogi Lanka Project of the SLMA. A unique feature in this guideline is that it addresses hitherto ill addressed issues in health care viz physical activity during pregnancy, dietary options and healthy lifestyle of the mother to be and her family, as well as universal screening of pregnant women. The international divide on screening methods and cut off values for blood glucose measurement has also been effectively addressed to ensure a pragmatic approach that takes into consideration regional specific issues. Web: http:/nirogilanka.org/pdf/final-gdm.pdf

${ }^{1}$ Consultant Community Physician, National Program Manager - Maternal Care, Family Health Bureau, Ministry of Health, Sri Lanka, ${ }^{2}$ Professor in Reproductive Medicine, Consultant Physician/Endocrinologist, Department of Obstetrics and Gynecology, Faculty of Medicine, University of Colombo, Sri Lanka. 


\section{Nirogi Maatha project -}

\section{Goal}

- To improve the quality of maternal diabetes care in Sri Lanka.

- To empower communities to be responsible for the prevention of diabetes and CVD commencing from pregnancy.

\section{Objectives}

- Capacity building of field staff managing pregnant women and children specifically on issues of GDM.

- Develop nationally relevant screening method for GDM and practice guidelines in accordance with international criteria.

\section{Target group}

- Field staff in maternal and child health in the state sector through the National Coordinating Centre and Focal Point, in the Ministry of Health - the Family Health Bureau.

\section{Intended project results}

- Capacity building of human and technical resources for screening, management and long term follow up of gestational diabetes at a national level spreading out to the grass root level.

\section{Planned activities to reach objectives}

- To develop training module and train field staff on holistic management of GDM/maternal diabetes during pregnancy and beyond.

- To develop IEC material on GDM and Hypertension during pregnancy.

\section{Expected outcomes}

- Capacity building of human and technical resources for screening, management and long term follow up of gestational diabetes at a national level.

- 150 trainers, 325 Medical officers of Health (MOH), 400 Health Nursing Sisters (PHNS) field midwives (PHM) will receive appropriate training on the core subject of GDM and its importance and will be enabled to deliver the necessary health education of women of reproductive age in Sri Lanka.

- Training module available for future training of MCH staff will ensure sustainability of projects.

- The childbearing female population and families of Sri Lanka will benefit.

The cooperation and commitment to practice guidelines and their implementation by all sectors of health care in Sri Lanka will be much appreciated. 